\title{
Glutamatergic mechanisms for speed control and network operation in the rodent locomotor CPG
}

\author{
Adolfo E. Talpalar* and Ole Kiehn* \\ Mammalian Locomotor Laboratory, Department of Neuroscience, Karolinska Institutet, Stockholm, Sweden
}

Edited by:

Eve Marder, Brandeis University, USA

Reviewed by:

Ronald L. Calabrese, Emory University,

USA

Joseph Fetcho, Cornell University, USA

${ }^{*}$ Correspondence:

Adolfo E. Talpalar and Ole Kiehn, Mammalian Locomotor Laboratory,

Department of Neuroscience, Retzius väg 8, Karolinska Institutet, 17177

Stockholm, Sweden.

e-mail: o.kiehn@ki.se and

adolfo.talpalar@ki.se
Locomotion is a fundamental motor act that, to a large degree, is controlled by central patterngenerating (CPG) networks in the spinal cord. Glutamate is thought to be responsible for most of the excitatory input to and the excitatory activity within the locomotor CPG. However, previous studies in mammals have produced conflicting results regarding the necessity and role of the different ionotropic glutamate receptors (GluRs) in the CPG function. Here, we use electrophysiological and pharmacological techniques in the in vitro neonatal mouse lumbar spinal cord to investigate the role of a broad range of ionotropic GluRs in the control of locomotor speed and intrinsic locomotor network function. We show that non-NMDA (non-NMDARs) and NMDA receptor (NMDAR) systems may independently mediate locomotor-like activity and that these receptors set different speeds of locomotorlike activity through mechanisms acting at various network levels. AMPA and kainate receptors are necessary for generating the highest locomotor frequencies. For coordination, NMDARs are more important than non-NMDARs for conveying the rhythmic signal from the network to the motor neurons during long-lasting and steady locomotor activity. This study reveals that a diversity of ionotropic GluRs tunes the network to perform at different locomotor speeds and provides multiple levels for potential regulation and plasticity.

Keywords: spinal cord, locomotion, central pattern generator, NMDA, 5HT, AMPA, kainate, NBQX

\section{INTRODUCTION}

Locomotion in mammals is a complex behavior entailing pacing of the rhythm, alternation of flexor-extensor muscles around different joints, and alternating activity between corresponding muscles on the left and right sides of the body. These properties are determined by the activity of central pattern generators (CPG) located in the spinal cord.

Glutamate transmission is thought to be essential for the excitatory activity to, and within the locomotor CPG (Beato et al., 1997; Nishimaru and Kudo, 2000; Whelan et al., 2000; Kiehn et al., 2008, 2010). At the level of the input to the CPG, glutamatergic neurons located in the hindbrain and descending to the spinal cord have been suggested by many to play an important role in initiating locomotion (Dubuc et al., 2008; Jordan et al., 2008; Roberts et al., 2008, Hägglund et al., 2010), although serotoninergic neurons have also been implicated (Schmidt and Jordan, 2000; Liu and Jordan, 2005). Increased firing frequency of these neurons is supposed to increase locomotor speed. Locomotor frequency has also been shown to be directly proportional to the concentration of glutamate or its agonists (Brodin et al., 1985; Cazalets et al., 1992) suggesting a strong glutamatergic speed control at the level of the input to the CPG. In lamprey, swimming frequency depends on the type of glutamate receptors (GluRs) activated indicating that NMDA- and non-NMDA receptor activation controls non-overlapping frequency ranges (Brodin et al., 1985; Alford and Grillner, 1990). Previous studies have conjectured that this relationship is not valid for mammals, since presumed nonNMDA and NMDA ligands produced similar effects on frequency, suggesting that GluRs controlled overlapping frequency ranges (Cazalets et al., 1992; Beato et al., 1997). In mammalian species, studies about glutamate regulation of the intrinsic operation of the CPG have concentrated on the need of different GluRs for rhythmgeneration (Kudo and Yamada, 1987; Beato et al., 1997; Nishimaru et al., 2000; Whelan et al., 2000; Cowley et al., 2005). These studies using pharmacological interaction with NMDA and non-NMDARs have provided conflicting results, indicating either that only nonNMDARs (Nishimaru et al., 2000; Whelan et al., 2000) or that none of the GluRs types themselves are essential for rhythm-generation (Beato et al., 1997; Cowley et al., 2005). Moreover, several reports show that quinoxaline ligands CNQX and DNQX may also block NMDARs (Birch et al., 1988; Kessler et al., 1989), challenging interpretations that assumed them to be selective non-NMDAR blockers (Cazalets et al., 1992; Douglas et al., 1993; Beato et al., 1997; Whelan et al., 2000). A recent study using Vglut2-knockout mice has even postulated that glutamate is completely dispensable for normal hindlimb CPG function (Wallen-Mackenzie et al., 2006).

The questions raised about the role of GluRs in locomotor rhythm-generation and coordination together with the dubious specificity of previously used non-NMDARs antagonists prompted us to study the role of GluRs in controlling locomotion in a broader perspective that includes the role of these receptors in the control of speed and intrinsic network function using specific pharmacological tools in the neonatal mouse preparation. We show that non-NMDA and NMDAR systems may independently mediate locomotor-like activity and that activation of these receptors sets different speeds of locomotor-like activity. Non-NMDARs intrinsic to the locomotor network are indispensable for achieving high locomotor speeds. NMDARs play a more important role than non-NMDARs in conveying the rhythmic signal within the 
network and to the motor neurons. The study reveals that selective activation of ionotropic GluRs is necessary for generating different locomotor speeds.

\section{MATERIALS AND METHODS PREPARATION}

Newborn C/57BL6 mice aged P0-P4 were used in all experiments. All experimental procedures followed the guidelines of the Swedish Animal Welfare Agency and were approved by the local animal care and ethical committee.

Mice were anesthetized with isoflurane before decapitation and evisceration. The hindbrain and the spinal cord were exposed ventral side up in a cold $4^{\circ} \mathrm{Clow} \mathrm{Ca}^{2+}$ Ringer's solution which contained in mM: $111 \mathrm{NaCl}, 3 \mathrm{KCl}, 11$ glucose, $25 \mathrm{NaHCO}_{3}, 3.7 \mathrm{MgSO}_{4}, 1.10$ $\mathrm{KH}_{2} \mathrm{PO}_{4}, 0.25 \mathrm{CaCl}_{2}$, and was bubbled with $95 \% \mathrm{O}_{2} / 5 \% \mathrm{CO}_{2}(\mathrm{pH}$ 7.4). Two different preparations were used for the electrophysiological experiments. When locomotor-like activity was initiated with drugs the spinal cord axis was cut at $\mathrm{C} 1$ and at S2-S3. In some experiments locomotor-like activity was evoked by electrical stimulation of the lower brainstem in which case the neural axis was cut rostrally between the mesencephalon and the diencephalon and caudally at S2-S3 (see later).

\section{ELECTROPHYSIOLOGICAL RECORDINGS}

The preparation was transferred to a recording chamber perfused with normal Ringer's solution containing in mM: $111 \mathrm{NaCl}, 3 \mathrm{KCl}$, 11 glucose, $25 \mathrm{NaHCO}_{3}, 1.25 \mathrm{MgSO}_{4}, 1.1 \mathrm{KH}_{2} \mathrm{PO}_{4}, 2.5 \mathrm{CaCl}_{2}$, bubbled with $95 \% \mathrm{O}_{2} / 5 \% \mathrm{CO}_{2}$ ( $\mathrm{pH} 7.4$ ). Recordings were obtained using suction electrodes attached to lumbar ventral roots (VRs) $\mathrm{L} 2$ and L5 on the left and right sides of the cord. In most cases two of the VR signals were AC recorded and band-pass filtered from $100 \mathrm{~Hz}$ to $1 \mathrm{kHz}$ (gain 20,000) while the two other VR signals were DC recorded using an Axoclamp-2A amplifier. The DC signals were then amplified 800 times using a custom-build DC amplifier and low-pass filtered at $20 \mathrm{~Hz}$. For analysis all the signals were digitally smoothed with moving averaging (window of $\pm 0.2 \mathrm{~s}$ ). AC recordings were full-wave-rectified in most cases. The main bursts in the L2 VR correspond to flexor activity while the L5 VR bursts correspond to extensor activity (Whelan et al., 2000). The extracellular signals were sampled at $1-2 \mathrm{kHz}$ using Axoscope (Molecular Devices). All recordings were made at constant room temperature of approximately $22^{\circ} \mathrm{C}$.

\section{DRUGS}

Locomotor-like activity was induced by bath application of different combinations of neuroactive drugs. In most of the experiments we used serotonin $(5 \mathrm{HT} ; 8 \mu \mathrm{M})$ in combination with dopamine (DA; $50 \mu \mathrm{M})$. The following glutamate receptor agonists were used in combination with 5HT and DA: $N$-methyl-D-aspartate (NMDA; 1-16 $\mu \mathrm{M}$ ) (Cazalets et al. 1992), D-glutamate (D-Glu) $(100-1000 \mu \mathrm{M})$, the non-NMDAR agonist kainate (KA, 0.3-2 $\mu \mathrm{M})$ (Cazalets et al. 1992), and the GluR5 kainate receptor agonist (RS)2-amino-3-(3-hydroxy-5-tert-butylisoxazol-4yl)propanoic acid (ATPA, 0.3-5 $\mu$ M; Tocris) (Procter et al., 1998; Kaminski et al., 2004; $\mathrm{Xu}$ et al., 2006). The following glutamate receptor antagonists were used: the broad spectrum non-NMDAR blocker, 2,3-Dioxo-6-nitro1,2,3,4-tetrahydrobenzo[f] quinoxaline-7-sulfonamide (NBQX, 10 $\mu \mathrm{M}$ ) (Menuz et al., 2007); the competitive NMDA receptor blocker, D-(-)-2-amino-5-phosphonopentanoic acid (AP5, 30-60 $\mu \mathrm{M}$ ) (Childs et al., 1988; Ornstein et al., 1989); the non-competitive NMDAR blocker (5S,10R)-( + )-5-methyl-10,11-dihydro-5Hdibenzo(a,d)cyclohepten-5,10-imine maleate (MK-801, $50 \mu \mathrm{M}$ Tocris) (Childs et al., 1988; Huettner and Bean, 1988); the GluR5subunit containing kainate receptor (KAR) blocker (S)-1-(2-amino2-carboxyethyl)-3-(2-carboxybenzyl)pyrimidine-2,4-dione (UBP 302; Tocris) dissolved in $10 \mathrm{mM} \mathrm{NaOH}$ (10 mM stock) for a working concentration of 1-12 $\mu \mathrm{M}$ (Mayer et al., 2006; Ireland et al., 2008; Wondolowski and Frerking, 2009); the AMPA receptor blocker 4-(8-Methyl-9H-1,3-dioxolo[4,5-h] [2,3] benzodiazepin-5-yl)benzenamine hydrochloride, (GYKI 52466 hydrochloride; Tocris) dissolved either in DMSO (stock $20 \mathrm{mM}$ ) or in $0.05-0.1 \mathrm{M} \mathrm{HCl}$ (stock $10 \mathrm{mM}$ ) for working concentrations of $20 \mu \mathrm{M}$ (Loscher and Honack, 1994; Micale et al., 2002). The concentrations of DMSO, $\mathrm{HCl}$ and other solvents used did not produce any effects by themselves on the locomotor-like activity. All of the drugs, if not otherwise specified, were purchased from Sigma.

\section{STIMULATION OF DESCENDING PATHWAYS}

Neural-evoked locomotor-like activity was elicited by electrical stimulation of the lower brainstem in preparations where the neural axis was cut rostrally between the mesencephalon and the diencephalon and caudally at S2-S3. Alternatively, locomotor-like activity was evoked from electrical stimulation at the midline in $\mathrm{C} 1-\mathrm{C} 2$ segments in preparations where the neural axis was cut at $\mathrm{C} 1$ and at S2-S3. We did not see any obvious difference in the locomotor-like activity induced by these two stimulation protocols. The electrical protocol for stimulation was similar to that employed previously by (Zaporozhets et al., 2004). Briefly, a large suction electrode (approximately $100 \mu \mathrm{M}$ in tip diameter) was used to deliver large amplitude (around $0.3-1 \mathrm{~mA}$ ), long duration (2-10 ms) and low frequency $(0.5-1 \mathrm{~Hz})$ stimuli. The total duration of each train of stimuli was usually 30-90 s.

\section{DATA ANALYSIS}

Data points for analyzing cycle periods and burst amplitudes were taken after the locomotor-like activity had stabilized, which was 10-15 min after the initial burst of activity. Locomotor-like activity was analyzed using rectified, smoothed and filtered AC signals and filtered DC signals of VR activity. The locomotor cycle period was calculated as the burst onset time in the $N$ 'th cycle to the burst onset time in the $N^{\prime}$ th +1 cycle. The burst amplitude (amplitude modulation) was calculated as the peak to trough normalized to the tonic level of activity in each trace (Kjaerulff and Kiehn, 1996). For the analysis of flexor/extensor coordination and left/ right coordination we selected 25 random locomotor cycles. The left (L) L2 was chosen as the reference trace while the right (R) L2, LL5, and RL5 were used as test traces (Kjaerulff and Kiehn, 1996). The onset of a locomotor burst in the reference trace corresponds to phase value of 0 in the circular plot while the onset of the next burst corresponds to the phase value 1. For the test trace, a phase value of 0.5 corresponds to alternation while a phase value of 1 (or 0 ) corresponds to synchrony. The individual phase values were analyzed in a circular plot where the mean timing of the 25 phase values in the test trace was calculated as a vector in the circular 
plot (Zar, 1974). The length of the vector reflects the concentration of the phase values around the mean. Rayleigh's test was used to determine if the mean phase values reached statistical significance with a $p$ value less than 0.05 . Coordination data are expressed by mean phases and phase deviations, which were calculated from the mean angle and its respective angular deviation in each set of experiments (Zar, 1974). Data are expressed as mean \pm standard error of the mean (SEM) if not otherwise specified.

\section{CHARACTERIZATION OF DELETIONS}

We used a series of criteria, mostly based on Lafreniere-Roula and McCrea (2005), to characterize deletions. If a deletion happened on one side of the cord, we called it a unilateral deletion while if it happened on two sides simultaneously we called it a bilateral deletion. Most of the observed deletions affected the active phase of the L2 VR burst, with or without simultaneous deletion of the corresponding inhibitory phase in the L5 VR burst. These events were classified as flexor deletions. We used a statistical test similar to the one used by (Lafreniere-Roula and McCrea, 2005) to determine whether deletions were resetting or non-resetting (an integer of the control period). This test is a special case of a $t$-test that compares whether a single specimen sampled at random (the period of the deletion, $Y_{1}$ ) belongs to a given population with a mean period $Y_{2}$ (Sokal and Rohlf, 1969). The $t$-statistic is calculated from the formula

$$
T=\frac{Y_{1}-Y_{2}-\left(\mu_{1}-\mu_{2}\right)}{s_{2} \sqrt{\frac{n_{2}+1}{n_{2}}}}
$$

where $Y_{1}$ is the single observation period, $Y_{2}$ is the mean period, $s_{2}$ is the sample standard deviation, and $n_{2}$ the sample size. $\mu_{1}$ and $\mu_{2}$ are the theoretical means that are the same. The degrees of freedom $(\mathrm{df})$ is $\mathrm{df}=n_{2}-1$. To estimate $Y_{2}$ we used a running average of five periods surrounding the deletion. If the deletion was close to 2 integers of the control period, the running average includes the sum of two periods. If the deletion was close to 3 integers of the control period the running average included the sum of three periods, etc. The number of cycles present in the deletion was estimated from plots of the periods as a function of time.

\section{RESULTS}

\section{NON-NMDA AND NMDA RECEPTORS ARE BOTH ACTIVE DURING NEURAL-EVOKED LOCOMOTOR-LIKE ACTIVITY}

Previous studies have shown that locomotor-like activity elicited by brainstem electrical stimulation (BES) in rats is blocked by bath application of either AP5 or CNQX (Cowley et al., 2005; Zaporozhets et al., 2006). However, the NMDAR blocking effect of CNQX (Birch et al., 1988; Kessler et al., 1989) prompted us to reexamine if both non-NMDARs and NMDARs were active during neural-evoked locomotor-like activity. For this purpose we electrically stimulated the caudal brainstem or the upper cervical spinal cord (Jordan, 1998; Jordan et al., 2008) to evoke a locomotor-like activity defined as left-right alternation seen at LL2 and RL2 (or LL5 and RL5), and alternation between the flexordominant L2 VR and the extensor-dominant L5 VR on the same side of the cord.
Application of $10 \mu \mathrm{M}$ NBQX completely blocked locomotorlike activity elicited by electrical stimulation in five of ten experiments, and partially blocked it in the other five (Figure 1A). NBQX exerted a maximal effect shortly after application and its effects were reversed after 20-30 min of washout $(n=10)$. In all the five experiments in which NBQX totally blocked locomotor-like activity, the regular rhythm and the alternating left-right and flexor-extensor pattern were disrupted, but barrages of uncoordinated activity did occur. In the five experiments in which NBQX only partially blocked the rhythmic locomotor-like activity, the barrages were shorter and the frequency of the rhythm reduced.

Blockade of NMDARs by bath application of AP5 $(30 \mu \mathrm{M})$ resulted in total blockade of electrically-evoked locomotor-like activity in six out of ten experiments and in severe disruption of coordinated bursts in the remaining four (Figure 1B). Typically, AP5 application rapidly and severely depressed the amplitude of the bursts, and prolonged washout totally reversed this effect. In the experiments showing incomplete blockade of activity, sporadic bursts occurred during the highest intensities and frequencies of the stimuli. Moreover, the preserved bursts often showed a pattern of coordination at various roots, suggesting that the NMDA component was neither indispensable for activating the CPG nor for coordinating activity within it.

These experiments showed that blockade of non-NMDARs and NMDARs either intrinsically to the CPG, at the drive, or at both may partly or even totally prevent locomotor-like activity from developing in response to electric stimulation of descending fibers. However, since the neural-evoked locomotor-like activity was blocked in most cases we could not determine in which of these components the receptors reside.

\section{LOCOMOTOR FREOUENCY IS DIFFERENTIALLY MODULATED BY THE ACTIVATION OF DIVERSE GLUTAMATE RECEPTORS IN THE MOUSE SPINAL CORD}

To be able to dissect the function of the diverse receptors in the CPG we set out to use drugs to generate locomotor-like activity with different frequencies. Fictive swimming frequency in the lamprey spinal cord is directly proportional to the concentrations of externally applied D-Glu (Cohen and Wallen, 1980), NMDAR or nonNMDAR agonists (Brodin and Grillner, 1985). However, the role of glutamate in frequency control has not been tested thoroughly in mammals, possibly because GluR agonists alone are not particularly efficient in inducing stable locomotor-like activity (Kudo and Yamada, 1987; Cazalets et al., 1992; Douglas et al., 1993). We therefore first asked if locomotor-like activity could be initiated by GluR agonists alone in the mouse spinal cord. We started using the natural neurotransmitter L-glutamate $(100-1000 \mu \mathrm{M})$, which bath applied elicited transient bursts of activity but no steady locomotorlike activity. The inability to induce steady locomotor-like activity by L-glutamate may be explained by its fast uptake and metabolism (Brodin and Grillner, 1985), leading us to test the more uptakeresistant stereoisomer D-Glu. However, application of D-Glu, NMDA or the broad spectrum non-NMDAR agonist kainate (KA) alone to the newborn mice spinal cord produced sustained but disorganized rhythmic activity (see also MacLean et al., 1998). Therefore, to obtain stable locomotor-like activity we combined bath application of NMDA/D-Glu/KA with constant concentrations of $5 \mathrm{HT}(8 \mu \mathrm{M})$ 
A

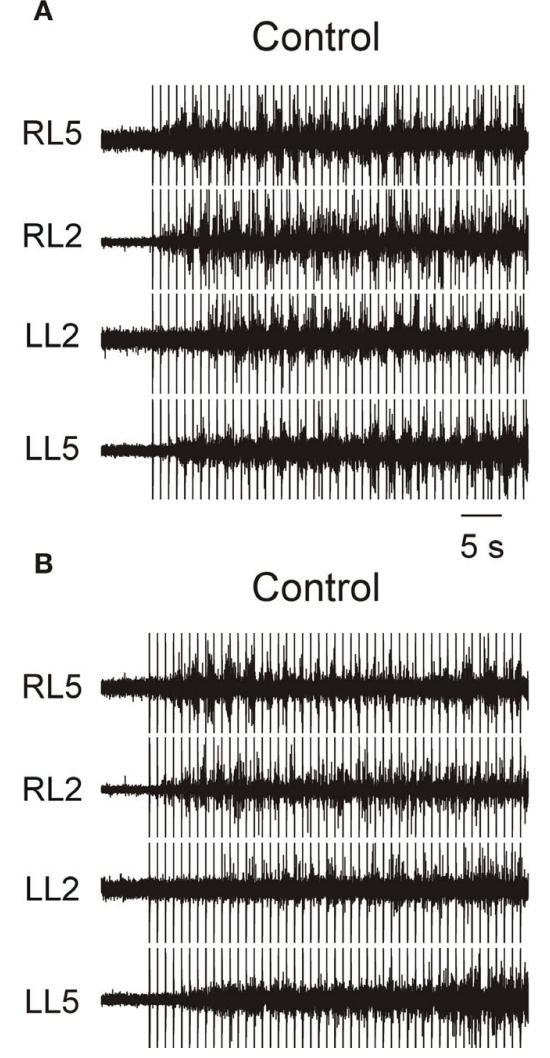

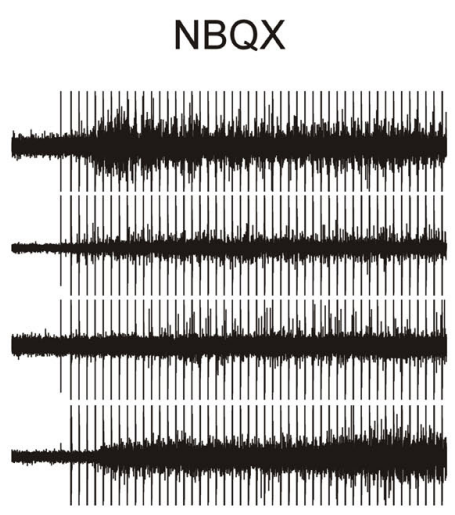

AP5

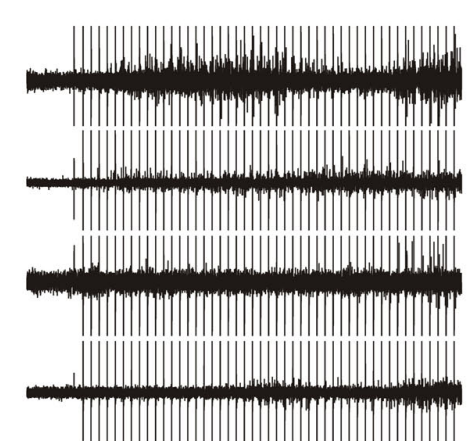

Washout
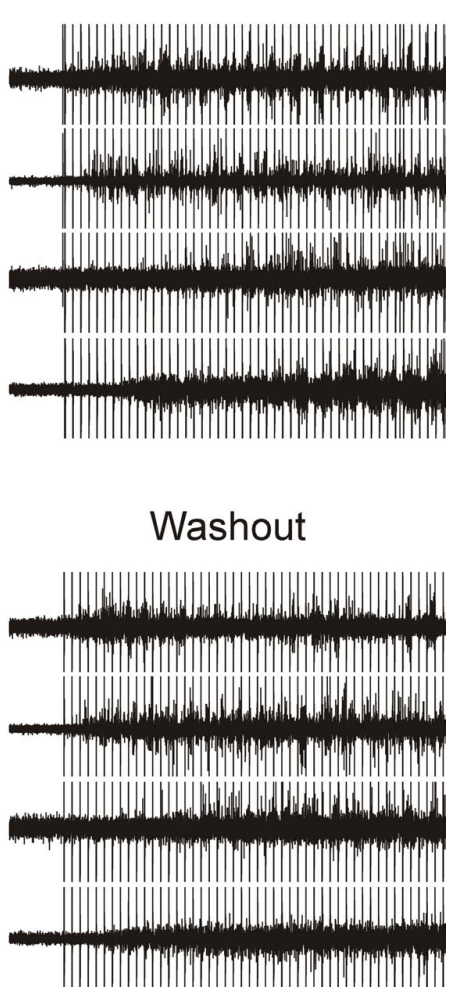

FIGURE 1 | Non-NMDA and NMDA receptors are important for neural-evoked locomotor-like activity. Stimulation frequency is $1 \mathrm{~Hz}$. All ventral root recordings are in $\mathrm{AC}$ mode. (A) Application of $10 \mu \mathrm{M}$ NBOX completely prevented or severely impaired the electrically-evoked locomotor-like activity in a reversible manner. (B) In the same preparation (as shown in A) application of $30 \mu \mathrm{M}$ AP5 completely or almost completely blocked the electrically-evoked locomotor-like activity in a reversible manner. and dopamine (DA; $50 \mu \mathrm{M})$ (MacLean et al., 1998; Jiang et al., 1999). The combination of 5HT and DA at these concentrations elicits slow locomotor-like activity with left-right (RL2/LL2 or RL5/LL5) and flexor-extensor (RL2/RL5 or LL2/LL5)alternation.

To assess glutamatergic influences in setting the locomotor frequency, we gradually changed the concentration of GluR agonists under conditions of constant concentrations of 5HT and DA.

Progressive adjustments in D-Glu concentration was directly reflected by changes in the locomotor frequency (Figure $2 \mathbf{A}$ ). The threshold concentration for inducing a significant change in the frequency was $100 \mu \mathrm{M}(n=4)$ while the maximal frequency elicited at these conditions was around $0.45-0.5 \mathrm{~Hz}$ (Figure 2C). The locomotor pattern observed at all frequencies during steady-state activity was normal with phase values for flexor-extensor (F-E) and leftright (L-R) coordination close to 0.5 (alternation) at the different D-Glu concentrations. The following mean phases and phase deviations were calculated for respective concentrations of $\mathrm{D}-\mathrm{Glu}$ (in $\mu \mathrm{M})$ : $100, \mathrm{~L}-\mathrm{R}=0.49 \pm 0.18 ; \mathrm{F}-\mathrm{E}=0.5 \pm 0.26 ; 200, \mathrm{~L}-\mathrm{R}=0.43 \pm 0.15$; $\mathrm{F}-\mathrm{E}=0.49 \pm 0.21 ; 300, \mathrm{~L}-\mathrm{R}=0.49 \pm 0.11 ; \mathrm{F}-\mathrm{E}=0.42 \pm 0.12$; and 500, $\mathrm{L}-\mathrm{R}=0.48 \pm 0.07 ; \mathrm{F}-\mathrm{E}=0.48 \pm 0.11$ ( $n=4$ for each condition $)$.

Application of NMDA in the presence of 5HT and DA produced a frequency/concentration curve roughly comparable to that of D-Glu (Figures 2B,C). Additional increases in D-Glu or
NMDA concentrations did not succeed in increasing the maximal frequency, but often resulted in a sudden reduction in burst amplitude and disruption of the rhythm. Plotting the amplitude of L2 VR bursts as a function of the glutamatergic agonist concentration resulted in both cases in inverted U-shaped curves displaying an initial increase in amplitude, until a maximum with low and medium concentrations, followed by a decrease in the amplitude at higher concentrations (Figure 2D). The dose of agonist that induced a decrease in bursts amplitude varied in different preparations (from 350 to $700 \mu \mathrm{M}$ for $\mathrm{D}-\mathrm{Glu}$ and $8-16 \mu \mathrm{M}$ for NMDA). Progressive adjustments in KA $(0.5-1.5 \mu \mathrm{M})$ on top of the standard concentrations of 5HT and DA induced locomotor-like activity that was often faster but less stable regarding the amplitude of bursts and less regular in period than that induced by NMDA or D-Glu (Figures 2E,F).

The frequency/concentration curve for KA was much steeper than that of D-Glu or NMDA under the same conditions (compare Figures 2C,F). This observation is consistent with previous results in lamprey (Brodin and Grillner, 1985) and rats (Cazalets et al., 1992). Consequently, the window for inducing stable locomotor activity with KA was narrower than that seen with D-Glu or NMDA. The threshold concentration with KA was around $0.25 \mu \mathrm{M}$ and the rhythm/pattern was disrupted at doses higher 


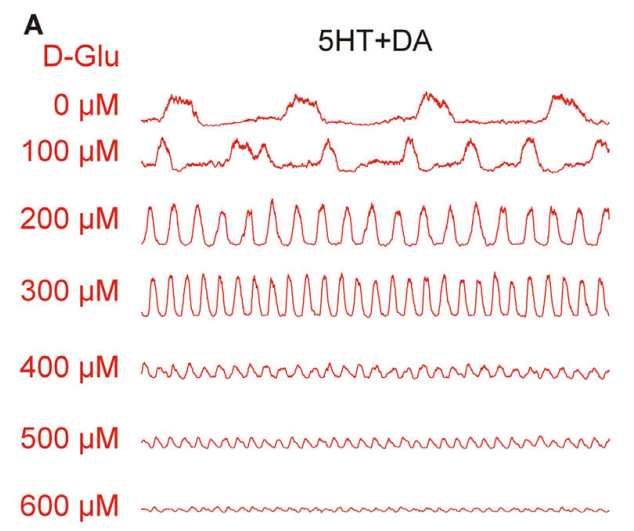

C

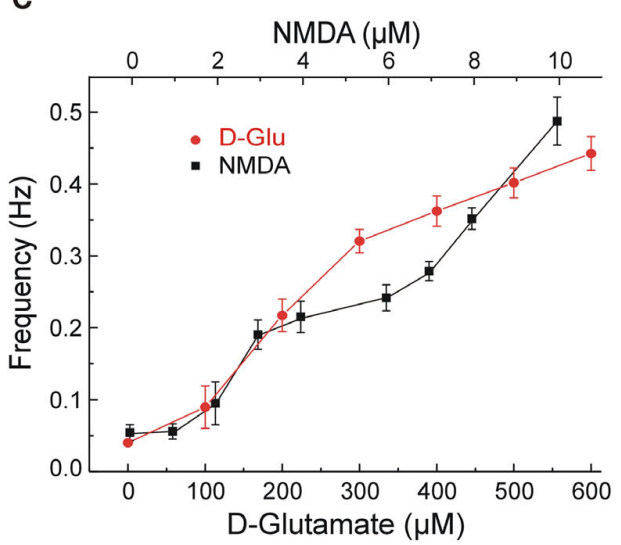

E

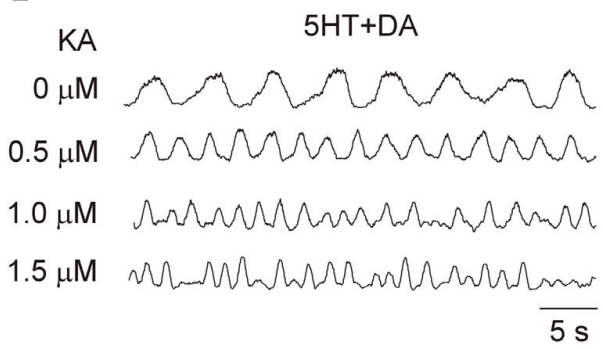

B

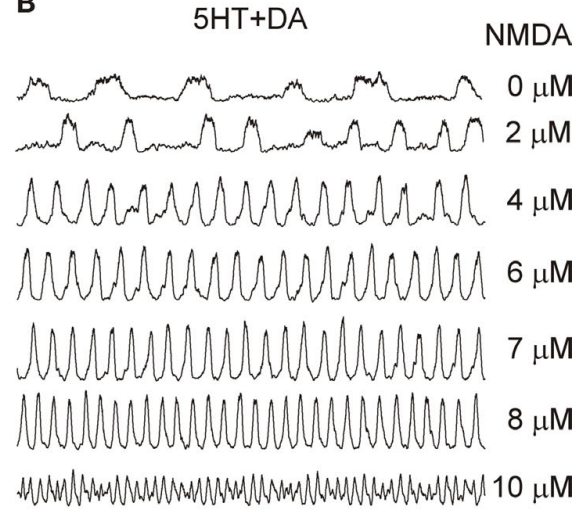

D

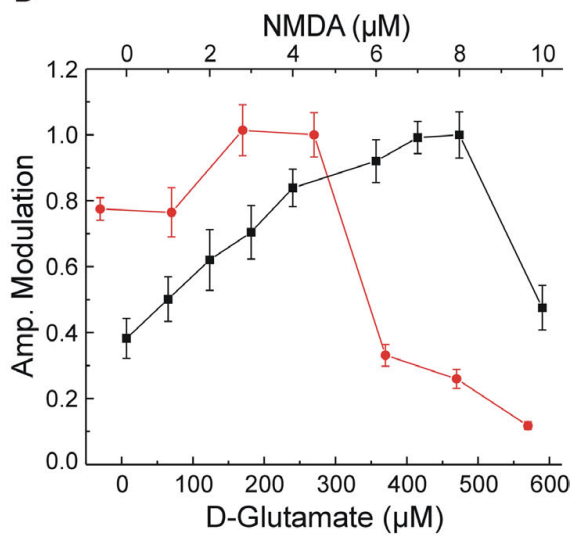

$\mathbf{F}$

G

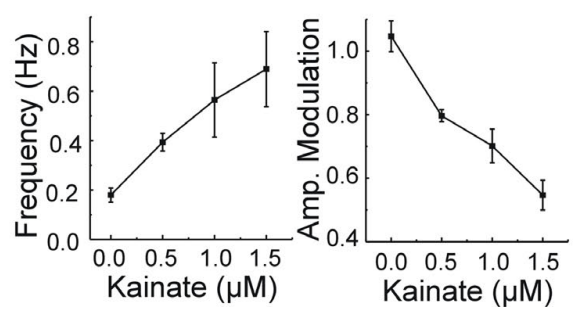

respect to the maximal amplitude). Graphs display means $\pm \mathrm{SD}$ ( $n=20$ each) Samples were obtained within 15 min of stable locomotor-like activity. (E) Variable concentrations of kainate (KA) applied on top of standard concentrations of $5 \mathrm{HT}$ and DA accelerated the locomotor rhythm. (F,G) The frequency of the locomotor rhythm increased as a function of $K A$ concentration (F) while the amplitude modulation of the L2 VR bursts (G) decreased as a function of KA concentration (normalized with respect to the maximal amplitude). than 2-2.5 $\mu \mathrm{M}$. The fastest KA-induced locomotor-like activity was characterized by a strong decrease in burst amplitude (Figures 2E,G). However, such locomotor-like activity had a normal average (around 0.5) left-right $(0.48 \pm 0.15$, mean phase and phase deviation, $n=7)$ and flexor-extensor $(0.45 \pm 0.12, n=13)$ coordination. The increase in amplitude variance that followed the increasing KA concentrations resulted in a change in mean amplitude that was inversely proportional to the KA concentration (Figure 2G).
The described experiments show that a progressively larger activation of GluR leads to an increase in the locomotor frequency with a complex effect on burst amplitude. D-Glu and NMDA induce a similar increase in frequency that is partially overlapping with that of KA. These results suggest a differential frequency control mediated by non-NMDARs and NMDARs. To dissect these mechanisms further, we performed experiments using pharmacological blockade that did not interfere with the drugs used to evoke the locomotor-like activity. Below we will refer to these drugs as the pharmacological drive. 


\section{PHARMACOLOGICAL DISSECTION OF GLURS INFLUENCES IN FREQUENCY CONTROL}

During locomotion GluRs may have the dual role of being activated by external inputs to the CPG (the "drive") and being mediators in its intrinsic synaptic transmission. Then, specific blockade of a group of receptors can produce a complex response because receptors at both of the levels are blocked. We attempted to separate these two functions in the in vitro system assuming that activation of the network (by a drive) leads to intrinsic release of glutamate (intrinsic function) that in turn contributes to the generation of locomotor-like activity. Then, we pharmacologically activated one of the GlutRs groups that we have defined above (see Locomotor Frequency is Differentially Modulated by the Activation of Diverse Glutamate Receptors in the Mouse Spinal Cord) to evoke locomotor-like activity (using a GluR-specific agonist), while we analyzed the intrinsic function of the network activity by applying antagonists of the other GluR group. For example, NMDA was used as a pharmacological drive for analyzing the intrinsic effects of non-NMDARs, and KA (and other non-NMDA agonists) was used to drive locomotor-like activity when analyzing the intrinsic NMDARs functions.

\section{Activation of non-NMDA receptors is essential for generation of high but not for low frequency locomotor-like activity}

When low concentrations on NMDA (below $7 \mu \mathrm{M}$ ), together with 5HT/DA, were used as a pharmacological drive, a low to medium locomotor frequency was induced (Figure 2B). Bath application of the broad spectrum non-NMDAR antagonist NBQX $(10 \mu \mathrm{M})$ during this locomotor-like activity produced a transient reduction of the locomotor frequency (increased period length) and a decrease in burst amplitude that lasted for about 2-5 min (Figures 3A,A1). The reduction in frequency reversed to control levels spontaneously after a short period ( $n=9$; Figures 3A,A1), suggesting that nonNMDARs within the network were active at the assessed frequency $(\sim 0.3$ to $0.2 \mathrm{~Hz})$. However, they were not essential for locomotorlike activity since the decelerating effect of NBQX rapidly disappeared. Raising the NMDA concentration (to 8-16 $\mu \mathrm{M}$ ) in the presence of standard 5HT-DA concentrations increased locomotor frequency to a maximum of about $0.45 \mathrm{~Hz}$.

For assessing the effect of NBQX on frequencies higher than $0.45 \mathrm{~Hz}$, we eliminated DA from the locomotor cocktail. This elimination allowed NMDA at concentrations of $10-16 \mu \mathrm{M}$, together with a constant $8 \mu \mathrm{M}$ concentration of $5 \mathrm{HT}$, to generate frequencies as high as $\sim 1 \mathrm{~Hz}$ (Kudo and Yamada, 1987). Under these conditions, NBQX produced a permanent reduction in locomotor frequency (Figures 3B,B1). After steady-state blockade by NBQX, coordination remained apparently within the normal range; left-right $(0.49 \pm 0.12, n=8)$ and flexor-extensor $(0.41 \pm 0.15, n=12)$.

Together the compiled data set showed that the effect of NBQX on frequency of the steady-state locomotor activity was not statistically significant when the control frequency was below $0.4 \mathrm{~Hz}$ (period $2.5 \mathrm{~s}$ ) ( $n=9$; Figure 3C, left), but there was a highly significant decrease in the frequency when the initial locomotor frequency was high ( $n=9, p<0.001$; Figure $3 \mathrm{C}$, right).

Plotting the difference in cycle period (\% change) as a function of the control cycle period resulted in an inverse linear relationship $(R=-0.85, p<0.0001, n=18$; Figure 3D) suggesting that the effect of NBQX was directly proportional to the control cycle period. Since the pharmacological drive (NMDA-5HT or NMDA/5HT-DA) was kept constant, these observations imply that with increasing frequency, there is greater activation of intrinsic network non-NMDARs in order to produce rhythmic activity. Independent of the control frequency, application of NBQX induced an early transient decrease in locomotor frequency that was $22 \pm 10 \%$ slower than the steady-state frequency obtained after full blockade ( $n=9, p<0.05$; Figures 3A1,B1). NBQX selectively reduced the amplitude of L2 VR bursts (by $39 \pm 6 \%$ compared to control, $n=9, p<0.005)$ at low frequencies $(<0.4 \mathrm{~Hz})$, but did not significantly affect the amplitude of L2 VR bursts at higher frequencies (Figures 3A1,B1). When the difference in L2 VR burst amplitude modulation (\% change) was plotted as a function of the control cycle period (Figure 3E), a significant inverse linear correlation $(R=-0.57, n=18, p<0.02)$ resulted. In contrast, NBQX did not significantly change the amplitude of L5 VR bursts, suggesting a preferential effect on more rostral lumbar segments.

All in all, the NBQX experiments show that intrinsic network non-NMDARs are indispensable for mediating locomotor-like activity at high frequencies but not at lower frequencies. The paradoxical transient effect observed after blocking non-NMDARs at low control frequencies suggests that network non-NMDARs are active during locomotor-like activity regardless of the frequency. However, their influences can be replaced completely or partially by other components of the network depending on the ongoing frequency.

AMPA receptors activation mediates high frequency locomotorlike activity. The variety of non-NMDAR types that may contribute to the NBQX-induced frequency-dependent slowing of the rhythm is vast, including subtypes of AMPA receptors (AMPARs) containing different composition of GluR1-4 subunits (Nagy et al., 2004) and KARs combining GluR5-7, KA-1 and KA-2 subunits (Jane et al., 2009).

The possibility of assessing specific effects of non-NMDARs using pharmacological means is restricted to the existence of highly selective blockers. GYKI $52466(20 \mu \mathrm{M})$ has been described as a high-affinity non-competitive antagonist of AMPARs (Dai et al., 2001). We therefore first assessed the effect of GYKI 52466 on high and medium/low frequency locomotor-like activity elicited using the same approach as in the last section, in order to determine the contribution of AMPARs activated by intrinsic glutamate release. Application of GYKI $52466(20 \mu \mathrm{M})$ did not significantly change the locomotor frequency when the control frequency was below $0.4 \mathrm{~Hz}$ (period $2.5 \mathrm{~s} ; n=8$; Figure 4B, left) but the drug was very efficient in reducing the locomotor frequency when applied during faster locomotor-like activity $(n=8, p<0.0001$; Figures 4A,B (right),C). The effect of GYKI 52466 on locomotor speed was, like for NBQX, frequency-dependent (Figure 4C). However, in addition to its steady effect NBQX also produced a transient decrease in locomotor frequency (Figures 3A1,B1) that was not seen with GYKI 52466 suggesting that the AMPAR function at high frequencies was not replaceable by other components in the network. Along with the reduction in frequency, GYKI 52466 application led to an increase in the amplitude of the VR bursts 
A

NMDA $7 \mu \mathrm{M}+5 \mathrm{HT}+\mathrm{DA}$ NBQX

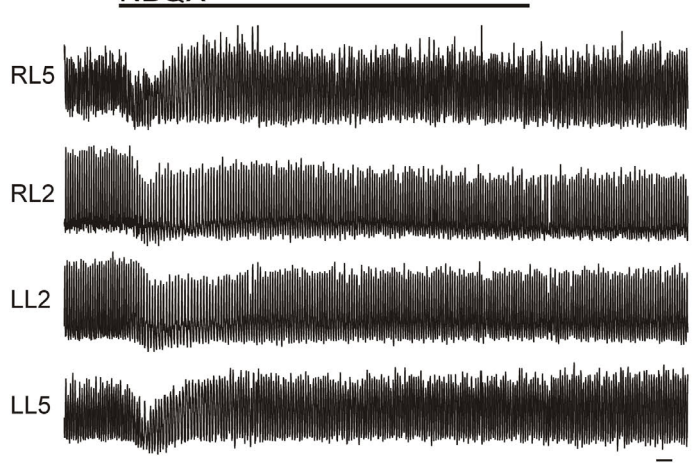

$3 \overline{0} \mathrm{~s}$

B $\mathrm{NMDA} 10 \mu \mathrm{M}+5 \mathrm{HT} 8 \mu \mathrm{M}$
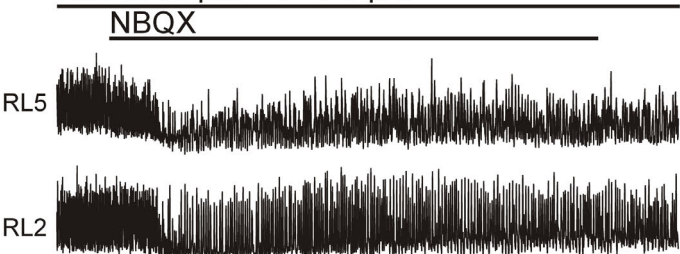

LL2

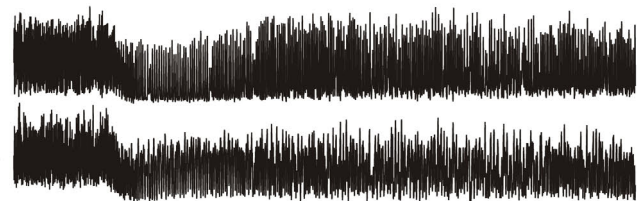

C

Period $>2.5 \mathrm{~s}$

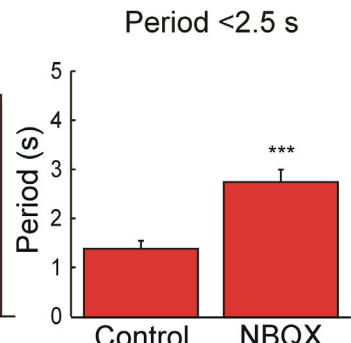

FIGURE 3 | Non-NMDA receptors in the lumbar CPG are necessary for high frequency locomotor-like activity. (A) NBQX produced a non-significant steady-state effect when the locomotor frequency was below $0.4 \mathrm{~Hz}$. VR activity was recorded in AC mode in RL2/RL5 and in DC mode in LL2/ LL5. (A1) Details of transient and steady-state effect of NBOX on ongoing locomotor-like activity. Red dashed lines mark the average peak amplitude of ventral root bursts and the tonic activity recorded in L2 before application of the antagonist. (B) Significant reduction of locomotor frequency by application of NBOX when the control frequency is higher than $0.4 \mathrm{~Hz}$. Same recording configuration as in (A). (B1) Details of transient and steady-state effects of NBOX, showing an initial larger reduction of frequency and amplitude of VR bursts (left). After some

by $49 \pm 11 \%(n=7, p<0.05$; Figures $4 A, D)$. This effect partially differs from the depressing effect of NBQX on the amplitude of the L2 VR burst at low frequency (Figure 3A1), suggesting that AMPARs are not the non-NMDARs mediating locomotor network connections at low frequency. Then, such function should be mediated by KARs.

\section{A1}

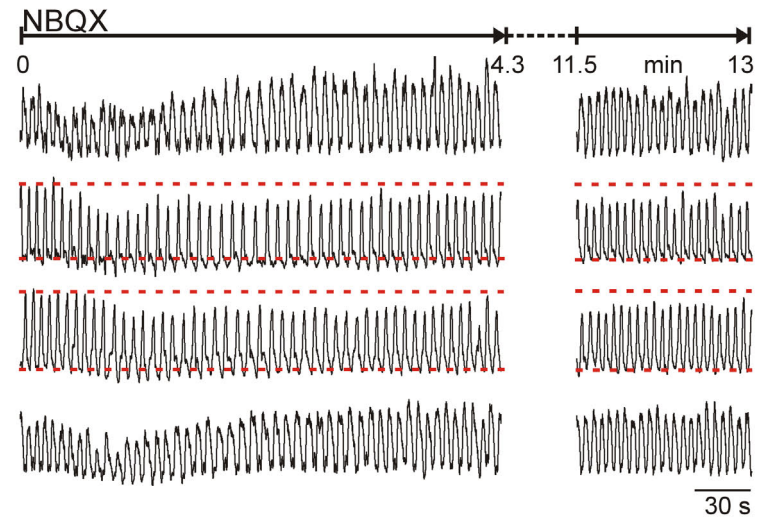

B1
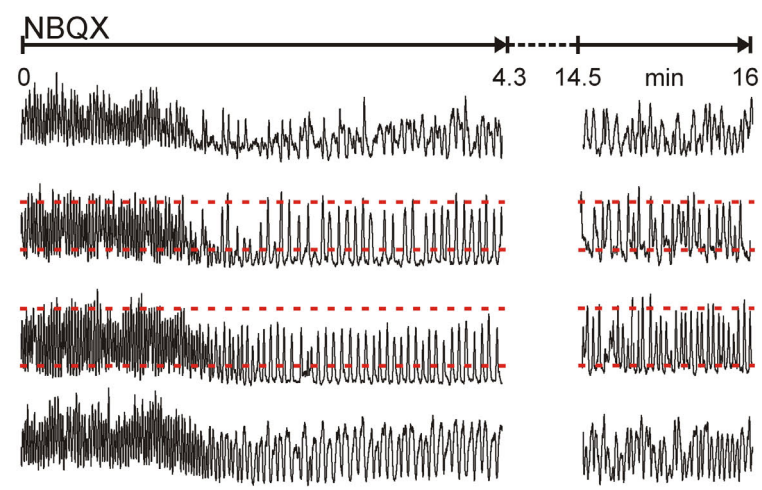

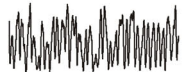

D
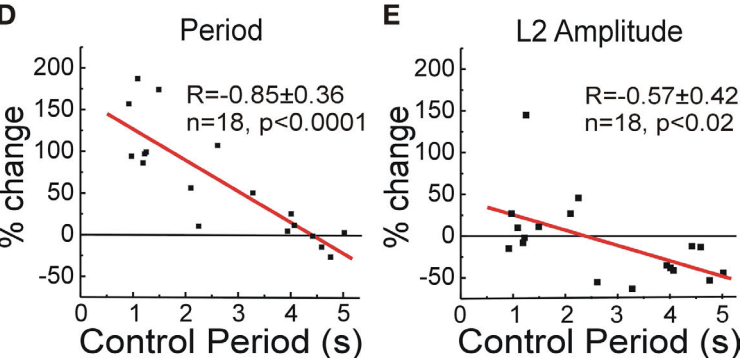

time there was a complete recovery of amplitude modulation but only partial recovery of frequency. (C) Statistical analysis of the effect of NBQX $(10 \mu \mathrm{M})$ on locomotor period (Means \pm SEMs, $n=9$ for each condition, paired $t$-test, ${ }^{* * *} p<0.001$ ). (D) Plot of the difference in steady-state locomotor period (\% change with respect to control) as a function of the control period showing a significant and linear inverse correlation for the effect of NBQX as a function of the control period $(n=18)$. (E) Correlation of NBQX-induced difference in amplitude modulation of the L2 VR bursts (\% of change with respect to control) as a function of the control period $(n=18)$ showing a significant reduction of the amplitude at frequencies $<0.4 \mathrm{~Hz}$ and lack of effect of NBOX at higher frequencies.

Kainate receptor activation contributes to speed regulation. Given that the effect of the AMPAR antagonist GYKI 52466 is relatively restricted to high locomotor frequencies and that the AMPAR/KAR blocker NBQX has a broader effect, other nonNMDAR types, in particular KARs, may be involved in speed control. 
A NMDA $16 \mu \mathrm{M}+5 \mathrm{HT} 8 \mu \mathrm{M}$ GYKI 52466

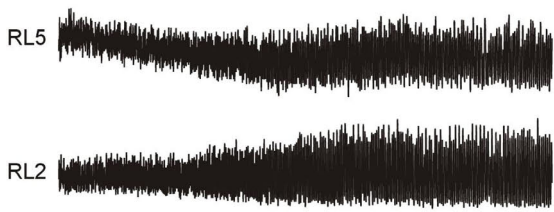

LL2
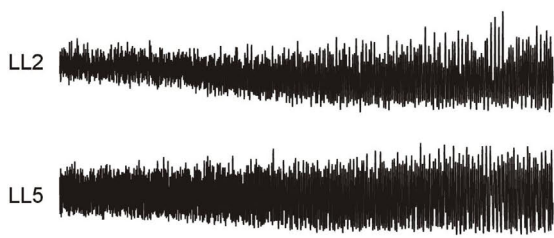

$\overline{30 s}$

B
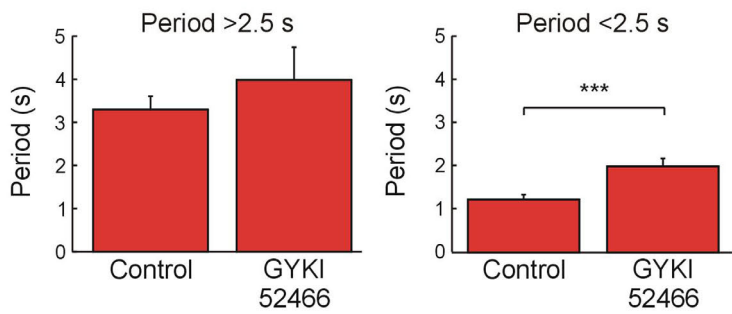

FIGURE 4 | AMPARs intrinsic to the network are necessary for generation of a high frequency locomotor-like activity. (A) Effect of GYKI $52466(20 \mu \mathrm{M})$ on locomotor-like activity at high frequency. VR activity was recorded in AC mode in RL2/RL5 and in DC mode in LL2/LL5. Expansions show VR activity before blockers (A1) and after full effect of GYKI 52466 (A2). (B) Statistical analysis of the effect of GYKI 52466 on moderate (left; $n=8$, not significantly different) and high locomotor frequencies (right, $n=8,{ }^{* *} p<0.001$ ). (C) Plot of the difference in locomotor
A1 $\quad$ A2

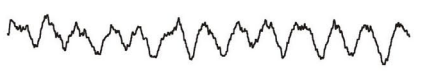

mummo

MMMMMW

Mummannm

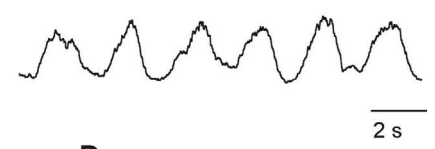

C
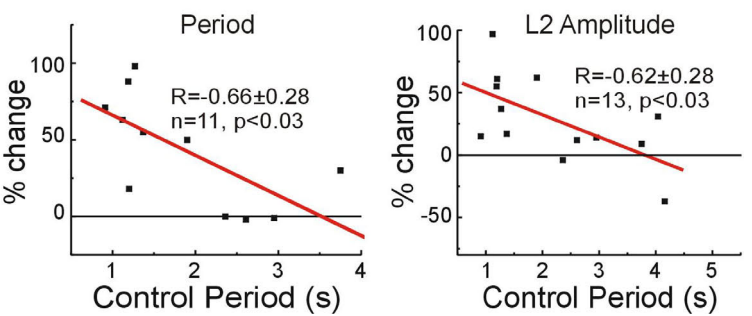

To investigate this proposition we used specific KAR ligands. UBP 302 is reported as an antagonist of KARs containing the GluR5 subunit (Clarke et al., 1997; Mayer et al., 2006; Ireland et al., 2008).

In order to evaluate the presence of GluR5s in the internal wiring of the locomotor network, UBP $302(5 \mu \mathrm{M})$ was applied during locomotor-like activity initiated by NMDA-5HT, with and without DA, at different frequencies. Like the other non-NMDAR blockers, the effect of UBP 302 showed frequency-dependence, producing a significant $34 \pm 13 \%$ increase in the locomotor period only when the frequency was higher than $\sim 0.6 \mathrm{~Hz}(n=6, p<0.005$; Figures 5A,B $)$. Although the amplitude of the bursts tended to increase after UBP 302 application, the trend did not reach statistical significance $(n=6)$. This lack of an effect on burst amplitude makes it unlikely that GluR5s plays a major role in synapses established between the CPG network and motor neurons.

In order to be sure that the blockade by UBP 302 was specific to GluR5s and was not overlapping with the targets of GYKI 52466, we compared their combined effects on locomotor-like activity induced by NMDA-5HT. Successive application of GYKI 52466 $(20 \mu \mathrm{M})$ in addition to the blockade induced by UBP $302(5 \mu \mathrm{M})$ resulted in an additional prolongation of the locomotor period by $41 \pm 4 \%(n=6, p<0.001$; Figures $5 \mathrm{C}, \mathrm{C1})$. Application of the period (as \% of change with respect to control) as function of the control period showing that the effect of GYKI 52466 was linearly proportional to the control locomotor period ( $R=-0.66, n=11, p<0.03$ ). (D) Plot of the amplitude modulation of the L2VR bursts (\% change with respect to control) as a function of the control period showing and inverse linear correlation $(R=-0.62, n=13$, $p<0.03$ indicating that GYKI 52466 increased the amplitude at high locomotor frequencies but had no effect at lower locomotor frequencies. broader spectrum non-NMDARs antagonist NBQX $(10 \mu \mathrm{M})$ on top of the previous two blockers, only marginally increased the period by another $13 \pm 3 \%(n=5, p<0.05)$.

The ability of UBP 302 to reduce the frequency only in a narrow frequency range (Figures 5A,B) compared to GYKI 52466 and NBQX, suggest that GluR5s mediate quantitatively few synaptic connections in the locomotor network. Therefore, other fractions of non-NMDARs (like KARs containing the GluR6-7, KA-1 and KA-2 subunits) are likely to play that role.

To determine if activation of GluR5-containing KARs may act as a drive, we induced low frequency locomotor-like activity with 5HT/DA alone and then added ATPA, a selective and highaffinity agonist of GluR5 KARs (Procter et al., 1998; Ireland et al., 2008). Addition of ATPA $(0.3-5 \mu \mathrm{M})$ instantaneously increased the frequency of the locomotor-like activity (Figures 5D,D1). This finding shows that the GluR5s may serve to accelerate the locomotor frequency.

Similar to KA, the window for the effect of ATPA was narrow. The threshold concentration for accelerating locomotor-like activity was around $0.3 \mu \mathrm{M}$. Higher concentrations such as $1 \mu \mathrm{M}$ increased the locomotor frequency from $0.09 \mathrm{~Hz}$ to $0.23 \mathrm{~Hz}(n=5$, $p<0.02$ ), while more than $2 \mu \mathrm{M}$ abolished the rhythm. The accelerating effect of ATPA was completely blocked by concomitant 


\begin{abstract}
A

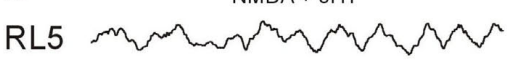

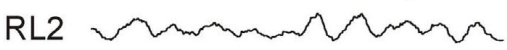

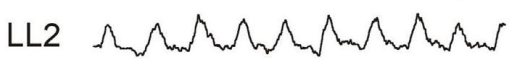

LL5 $w M M M M M M M M M$.
\end{abstract}

C

$\mathrm{NMDA}+5 \mathrm{HT}$

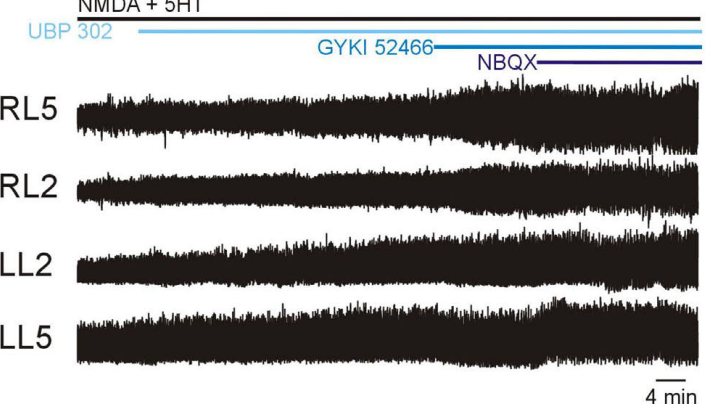

D

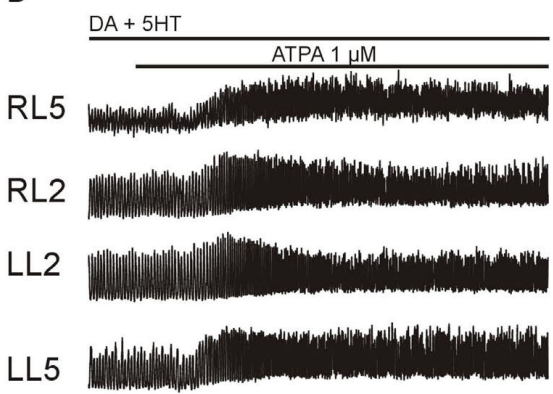

D1
E DA + 5HT + ATPA $1 \mu M$
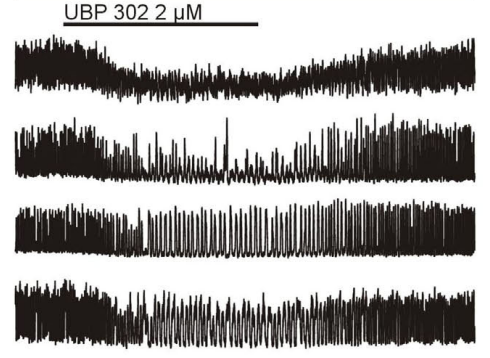

E1

$D A+5 H T+A T P A+$ UBP 302

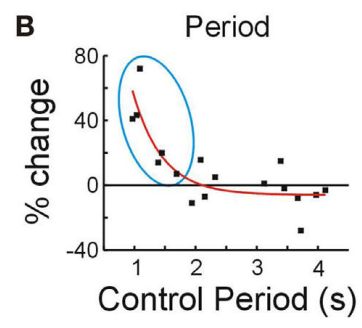

C1

$1 \mathrm{~s}$

+UBP $302+$ +GYKI 52466

$+\mathrm{NBQX}$

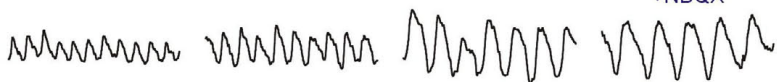

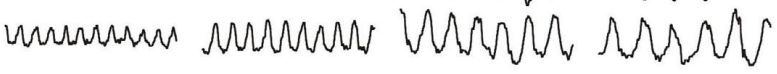

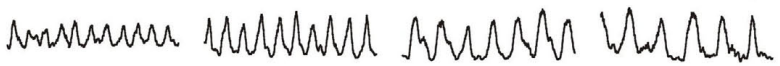

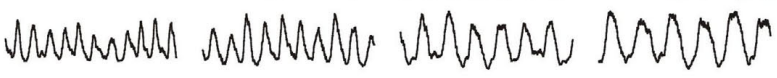

$F$

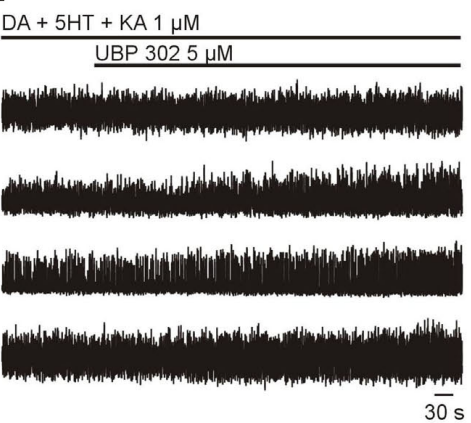

F1

$\mathrm{DA}+5 \mathrm{HT}+\mathrm{KA}+\mathrm{UBP} 302$ $\overline{2.5 \mathrm{~s}}$

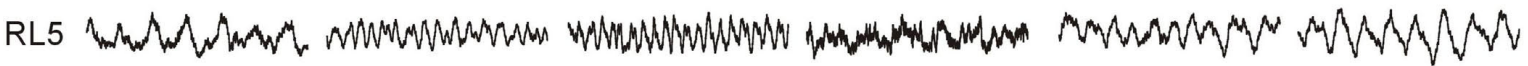

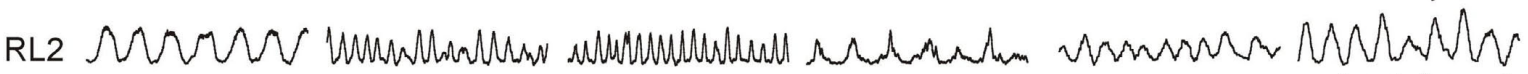

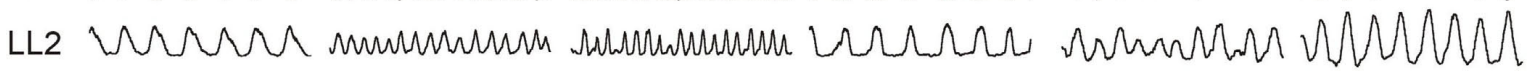

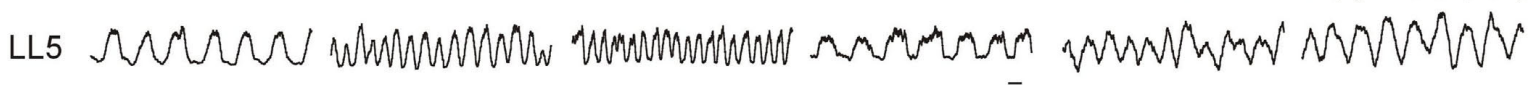
2s

FIGURE 5 | Kainate receptor activation contributes to speed regulation. VR activity was recorded in AC mode for RL2/RL5 and in DC mode for LL2/ LL5. (A) High frequency locomotor-like activity elicited by NMDA-5HT was reduced in frequency by UBP $302(5 \mu \mathrm{M})$. (B) Plot of the UBP 302-induced difference in the locomotor period (\% change with respect to control) as a function of the control period. Note the significant increase in the period at high frequencies (control frequency $>0.6 \mathrm{~Hz}$; blue ellipse; $n=6$,

$\left.{ }^{* * *} p<0.005\right)$, but the lack of effects at lower frequencies $(n=11)$. (C) Cumulative effect of different non-NMDAR antagonists, UBP $302(5 \mu \mathrm{M})$, GYKI $52466(20 \mu \mathrm{M})$ and NBOX (10 $\mu \mathrm{M})$ on 5HT/NMDA induced locomotor-like activity. The VR recording configuration was as in (A). (C1) Details of the effects of non-NMDAR blockade on bursts amplitude and frequency of locomotor-like activity. (D) Activation of GluR5s increased the locomotor frequency. ATPA $(0.3-2 \mu \mathrm{M})$, a specific GluR5 KAR agonist, increased the frequency of locomotor-like activity initiated by standard concentrations of 5HT-DA, $n=5, p<0.02$. (D1) Expansions show VR activity before and after full effect of ATPA. (E) ATPA-mediated acceleration of the locomotor rhythm was specifically blocked by bath application of the GluR5 antagonist UBP-302 (1-3 $\mu \mathrm{M})$. (E1) Expansions show VR activity before and after full effect of UBP 302. (F) Bath application of UBP 302, at even higher doses than used to suppress ATPA effects $(5-12 \mu \mathrm{M})$ only partially reduces the frequency of the locomotor-like activity initiated by the broad spectrum non-NMDAR agonist KA (together with the standard concentrations of 5HT-DA). (F1) Expansion shows VR activity before and after full effect of UBP 302. application of 1-2 $\mu \mathrm{M}$ UBP 302 (Figures 5E,E1; $n=5, p<0.05$ ), suggesting that these effects resulted from selective activation and blockade of GluR 5 s.
The high potency of ATPA and its specific blockade by UBP 302 show the efficacy of GluR5s for inducing locomotor rhythm. These effects coincide with the ability of KA and D-Glu to serve 
as locomotor drives (Figure 2). These results suggest that a nonNMDAR drive may be mediated by GluR5s or by other pharmacology undetermined non-NMDARs (e.g., receptors containing GluR6, GluR7, KA1, KA2 subunits or their combinations). In order to explore this possibility further, we initiated locomotor-like activity with KA, which acts as a broad spectrum non-NMDAR agonist activating AMPARs and almost all KARs, and evaluated its role at the level of the drive. During locomotor-like activity initiated by KA in combination with standard concentrations of 5HT-DA, bath application UBP 302 at ATPA-antagonizing concentrations (1-2 $\mu \mathrm{M})$ or higher $(5-12 \mu \mathrm{M})$ produced a much smaller reduction in the frequency compared to the reduction that it produced when locomotor-like activity was initiated by ATPA-5HT-DA (Figures 5F,F1). This finding demonstrates that GluR5s do not have a predominant role at the level of the locomotor drive and that the non-NMDAR component is probably mediated by a combina- tion of pharmacology undetermined receptors including AMPARs and/or other KARs (e.g., receptors containing GluR6, GluR7, KA1, KA2 subunits or their potential combinations).

\section{NMDA RECEPTORS HAVE LITTLE INFLUENCE ON INTRINSIC HIGH FREQUENCY SPEED CONTROL}

To study the role of NMDARs in speed control we used either KA or ATPA, in combination with 5HT and DA, to evoke fast (above $0.4 \mathrm{~Hz}$ ) locomotor-like activity and then blocked the intrinsic NMDAR component.

Blockade of NMDARs by AP5 or MK-801 during locomotor-like activity elicited by KA-5HT-DA resulted in a relatively constant high frequency locomotor-like activity (Figure 6A), partial reduction in the amplitude of the bursts and a high occurrence of deletions in flexor-related bursts either unilaterally or bilaterally (RL2 and LL2; Figures 6 A1,A2). These effects were produced by application of
A

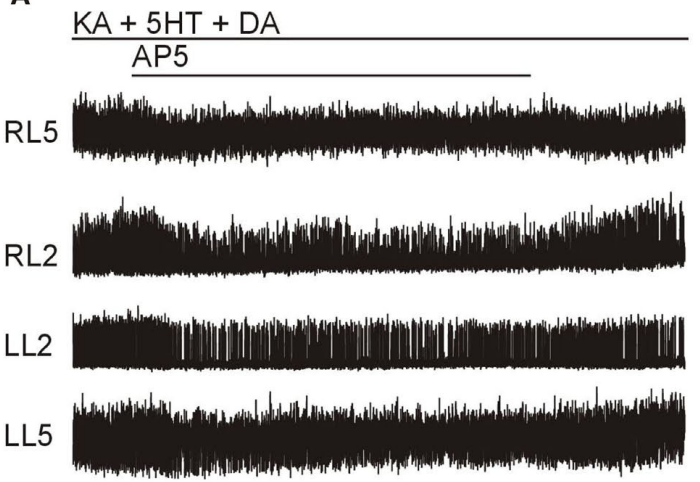

$6 \overline{0} \mathrm{~s}$
A1

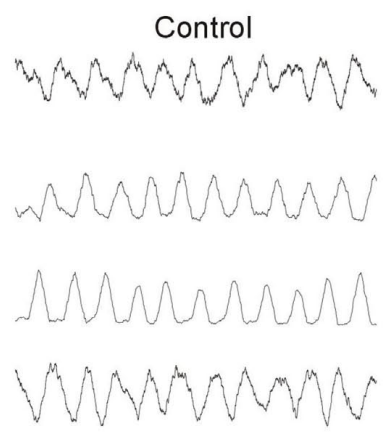

\section{A2}

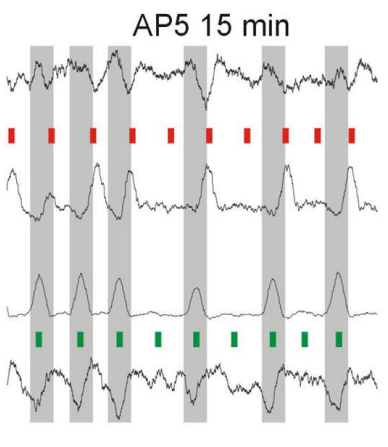

2s

A3

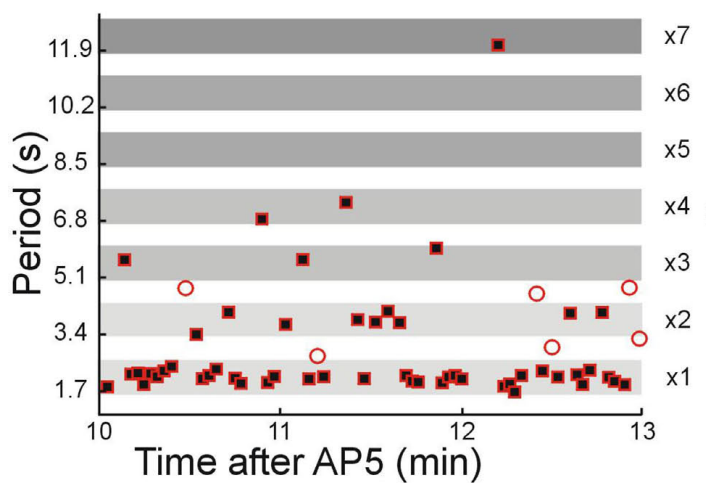

FIGURE 6 | NMDARs have a small effect on frequency control but contribute to the transmission of locomotor signal to motor neurons. (A) Application of AP5 $(30 \mu \mathrm{M})$ during locomotor-like activity elicited by KA-5HT-DA led to non-resetting flexor deletions with an unchanged frequency of the rhythm and preserved left-right and flexor-extensor coordination on the spared bursts. VR activity was recorded in AC mode for RL2/RL5 and in DC mode for LL2/LL5. (A1,A2) Expansion of VR recordings from (A) in control conditions (A1) and after 15 min of AP5 application (A2). Note that despite the occurrence of bilateral flexor deletions, the frequency of the rhythm [red bars right flexors (RL2); green bars left flexors (LL2)], the left-right alternation (large gray bars) and the flexor-extensor alternation (red
B

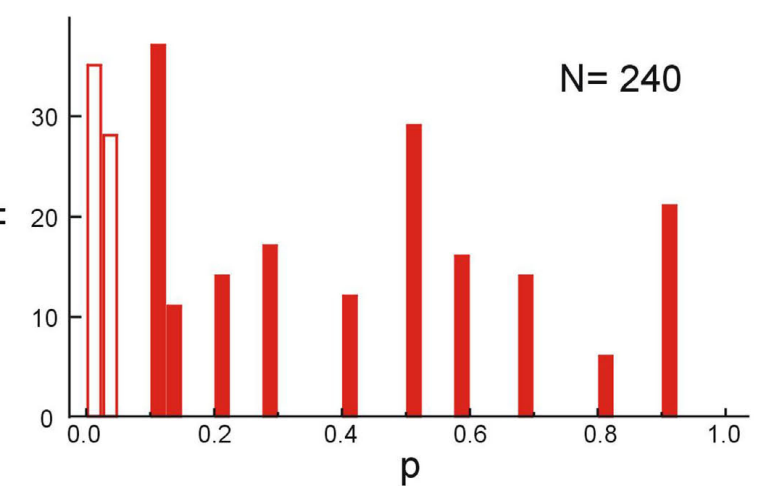

and green bars) are conserved in the preserved bursts. (A3) Plot of the instantaneous locomotor period of bursts as measured in the L2 ventral root during an epoch of 3 min of steady-state effect of AP5 $(30 \mu \mathrm{M})$. The occurrence of non-resetting flexor deletions is seen as integer multiples of the basal locomotor period (the width of the period bands was set to fit the maximum and minimum values of the basal period in the plotted epoch). Empty circles show periods between bursts that did not fit the criteria for non-resetting deletions. (B) Frequency histogram showing the proportion of deletions that fit the criteria for non-resetting deletion (filled bars; $p>0.05$; $n=177, \sim 75 \%$ ) and for resetting deletion (empty bars; $p<0.05$; $n=63, \sim 25 \%$ ). 
$30 \mu \mathrm{M}$ of AP5 $(n=9)$, not changed by increasing AP5 concentration to $60 \mu \mathrm{M}(n=6)$ and reversed after washout. Moreover, the phenomena were reproduced by application of the selective and non-competitive NMDAR blocker MK-801 (50 $\mu \mathrm{M} ; n=3)$.

The flexor deletions induced by NMDAR blockade were followed by tonic activity in extensor-dominated roots (RL5 and LL5), showing a reduction or absence of the corresponding inhibitory component that occurs concomitant to the ipsilateral flexor bursts (Figure 6A2; red and green short bars). Many of these deletions resemble the so-called non-resetting flexor deletions (Figure 6A3) that have been previously described in the cat spinal cord during brainstem-induced locomotor activity (Lafreniere-Roula and McCrea, 2005) with the exception that in the mouse the deletions could happen either unilaterally or bilaterally.

To verify the nature of the deletions we quantified the deletions in seven animals before and after NMDAR blockade using a statistical test to determine whether the resulting period during the deletion was an integer (non-resetting) or was not an integer (resetting) of the control cycle period (see Materials and Methods). This test showed that 177 out of 240 deletions were non-resetting while the remaining 63 qualified as resetting deletions (Figure 6B).

In 2 of 9 experiments, the nature and number of potential deletions could not be evaluated because the AP5-induced perturbations of the rhythm were continuous (without the conserved epochs of control frequency) leading to an apparent constant reduction of the frequency. Thus, the remnant of locomotor-like activity, following NMDAR blockade, is entirely mediated by non-NMDARs.

\section{DISCUSSION}

In the present study we investigated the role of ionotropic GluRs in the locomotor network function using specific pharmacological tools in the neonatal mouse in vitro preparation. We show that non-NMDAR and NMDAR systems may independently mediate locomotor-like activity and that non-NMDAR and NMDAR activation set different speeds of drug-induced locomotor-like activity. Our study suggests diverse roles of the two receptor families in generating locomotion at various network levels.

\section{SPEED CONTROL OF LOCOMOTOR-LIKE ACTIVITY}

Locomotion is ultimately determined by the frequency and the pattern generated by the spinal CPG. The glutamatergic control of speed described in this study operates without significantly changing the typical pattern of left-right and flexor-extensor coordination that characterizes locomotor-like activity in the in vitro neonatal mouse preparation, thereby defining mechanisms of modulation of a single behavior, rather than a switch to a more speed-efficient gait which involves major changes in muscle coordination (Gillis and Biewener, 2001). Lack of appreciable change in the flexor-extensor and left-right alternating pattern observed in the VR recordings does not exclude potential changes in muscle coordination which are not detected by VR recordings. In vertebrates, a simple form of speed control, not requiring modification of the locomotor pattern, occurs when the frequency of the rhythm increases proportionally to the external glutamatergic drive (Brodin and Grillner, 1985; Alford and Grillner, 1990; Cazalets et al., 1992). Here, we show that speed of locomotor-like activity in vitro can be controlled by NMDARs and non-NMDARs that exert regulation at what appear to be various levels of the locomotor network. These effects were revealed using pharmacological protocols that allowed us to separately block NMDARs and non-NMDARs activated by glutamate intrinsically released by the network in the presence of a constant pharmacological drive, in a wide range of locomotor frequencies. To discriminate these effects, we used direct agonist activation of the in vitro locomotor network combined with selective receptor blockade of the effects mediated by intrinsic glutamate release.

Activation of both the NMDA and non-NMDAR families seem to have ability to pace the locomotor rhythm suggesting that nonNMDARs and NMDARs maintain such interactions during the release of glutamate from descending fibers. Results obtained during electrical stimulation of the descending fibers (Figure 1) may support this conclusion. The contribution from non-NMDARs is mediated to a large degree by KARs, containing GluR5 and probably GluR6/7 and KA1/KA2 subunits, together with a small contribution from AMPARs. The variety of KARs that are able to pace the rhythm may be critical because AMPARs show strong desensitization when exposed to prolonged tonic glutamate activity, which in a physiological context may happen when the locomotor frequency is increased by a stronger descending drive. A combination of receptors acting as drive may, therefore, safeguard a stable speed control at the level of the drive.

Non-NMDARs and NMDARs intrinsic to the locomotor network seem to have specific roles in speed control. Thus, non-NMDARs, including both AMPARs and GluR5 KARs, are indispensable for achieving the highest locomotor frequencies, with little or no contribution from NMDARs. At lower frequencies NMDAR activation and probably KARs containing the GluR6/7 or KA1-KA2 subunits contribute to the speed control. These findings suggest that the site for intrinsic speed control is probably at the excitatory synapses between CPG neurons. Since CPG neurons are rhythmically active (Kiehn et al., 1996; Butt and Kiehn, 2003; Zhong et al., 2006; Quinlan and Kiehn, 2007; Tazerart et al., 2008) with phases of excitation and inhibition, intrinsic AMPARs will be less prone to desensitization. Non-NMDAR kinetics can be more than 10 times faster than NMDAR kinetics (Edmonds et al., 1995). Faster kinetics may allow faster cycling during activation at frequencies due to less receptor occupancy and saturation. Together, our results suggest a direct relationship between the specific kinetics of the receptors and their role in the intrinsic speed control.

The findings that the locomotor network can operate with NMDARs alone may seem to contradict previous studies in the neonatal mouse, which showed that slow locomotor rhythms initiated by 5 HT-DA or $5 \mathrm{HT}$ were not suppressed by AP 5 but only by CNQX or kyruneate (Nishimaru et al., 2000; Whelan et al., 2000). The transient reduction in the frequency by blockade of broad non-NMDARs blockers and the spontaneous recovery and maintenance of the activity by remnant network components suggests that non-NMDARs and NMDARs are both active at low frequencies (Figures 3A,A1). The nature of NBQX transient effects is unknown but bear resemblance to homeostatic changes that have been described in hippocampal cells where chronic NBQX exposure led to up-regulation of receptors containing the GluR1 subunit (Thiagarajan et al., 2005), to increase in synaptic strength (Murthy et al., 2001) or to activity changes leading to modification of NMDAR trafficking and composition (Perez-Otano and Ehlers, 2005). We suggest that the recovery in 
frequency is due to an up-regulation of NMDA receptors. It is noteworthy that other studies in the neonatal rat showed that NMDAR blockade could suppress the rhythm at low (Beato et al., 1997; Cowley et al., 2005) but not at higher locomotor frequencies (Cowley et al., 2005). Changes in the active proportion of non-NMDARs/NMDARs may explain these apparent divergences in the results.

The need of glutamate, and even of specific GluRs, for rhythmgeneration is in conflict with the generation of drug-induced rhythmic activity under conditions of suppressed spinal glutamatergic transmission in a Vglut2 knockout mouse (Wallen-Mackenzie et al., 2006). The present experiments argue that the drug-induced rhythmic activity elicited in Vglut 2 knockout mice may be generated by network mechanisms different from those found in wild-type mice, for example by a predominantly inhibitory network.

\section{SPECIFIC ROLES OF GLUTAMATERGIC TRANSMISSION IN PATTERN GENERATION AND CPG-TO-MOTOR NEURON TRANSMISSION}

Coordination of left-right and flexor-extensor alternation in the rodent CPG is exerted by inhibitory neurons (Cazalets et al., 1995; Cowley and Schmidt, 1995; Butt and Kiehn, 2003; Lanuza et al., 2004; Hinckley et al., 2005; Kiehn, 2006; Quinlan and Kiehn, 2007). The blockade of different GluRs changes locomotor frequency, and therefore rhythm-generation without affecting leftright and flexor-extensor alternation (unless the rhythm is severely depressed). The conservation of pattern at various speeds indicates a precise tuning of inhibition to changes in locomotor speed, suggesting a similar speed-related utilization of kinetically suitable GluRs for the activation of inhibitory interneurons involved in pattern-generation as those involved in rhythm-generation.

Blockade of NMDARs and non-NMDARs also affected the amplitude of the bursts seen in VR recordings. At high locomotor frequencies blockade of all non-NMDARs decreased the frequency without affecting the amplitude (Figures 3B1,E) while blockade of non-NMDARs during low frequency locomotor-like activity specifically decreased the amplitude of flexor bursts (L2 VRs; Figures 3A1,E) an effect that appeared to be mediated by KARs. These results show that non-NMDARs play a strong role for CPG-to-motor neuron coupling. The effect of these changes in amplitude could be either at the motor neuron level, or up-stream from last-order interneurons.

Blocking NMDARs produced unilateral and bilateral deletions that were either resetting or non-resetting (Figure 6). The deletions show similarities to what has been reported for brainstem locomotor activity in the cat (Lafreniere-Roula and McCrea, 2005). The resetting deletions $(\sim 25 \%)$ were less frequent than the non-resetting deletions $(\sim 75 \%)$. The resetting deletions may be consequence of increased variance in the cycle duration, while the non-resetting deletions may result from failure of transmission of the rhythmic signal to the motor

\section{REFERENCES}

Alford, S., and Grillner, S. (1990). CNQX and DNQX block non-NMDA synaptic transmission but not NMDAevoked locomotion in lamprey spinal cord. Brain Res. 506, 297-302.

Beato, M., Bracci, E., and Nistri, A. (1997). Contribution of NMDA and non-NMDA glutamate receptors to

neurons. In the case of the non-resetting deletions the failure of transmission involves rhythmic excitation in flexors (L2 VRs), inhibition in extensors (L5 VRs), and their corresponding phases across the cord. Structurally, such dissociation is most easily explained by a two or three layered CPG where the rhythm-generating layer is separated from the pattern-generating layer (Lafreniere-Roula and McCrea, 2005; Endo and Kiehn, 2008; McCrea and Rybak, 2008).

These experiments suggest that there are NMDA-dependent synaptic inputs coordinating the rhythm-generating layer with the interconnected pattern-generating units of segmental (e.g., excitatory drive of flexors in L2) and intersegmental (reciprocal inhibition between L2 and L5) levels as well as between the left and right side of the cord (bilateral flexor deletions). Thus, for the bilateral non-resetting flexor deletions to occur simultaneously at four roots, the last-order excitatory interneurons must be tightly connected to activation of inhibitory interneurons onto antagonistic motor neuron pools on the same side of the cord (see Figure 8 in Endo and Kiehn 2008) and to reciprocal connections between the two sides of the cord.

\section{INTRINSIC IONOTROPIC GLUTAMATE RECEPTORS: A SITE FOR PLASTICITY AND MODULATION OF THE NETWORK FUNCTION}

The intrinsic need for non-NMDARs to generate high speed locomotor-like activity and the need for NMDARs for reliable transmission within the CPG network provide at least two targets for intrinsic network modulation (Katz and Frost, 1996; El Manira et al., 2008) and plasticity that may change as a consequence of the ongoing network activity. For example it has been shown in the lamprey spinal cord that NMDAR function can be enhanced by intrinsic network mediated activity via glutamate activation of mGluR1 receptors (Krieger et al., 2000; Nanou et al., 2009). AMPARs effects may also be scaled instantly by synaptically induced $\mathrm{Na}^{+}$transients (Nanou et al., 2008). Fast modulation of the balance between the non-NMDARs and NMDARs was in fact seen in the present experiments when all non-NMDARs were blocked (Figure 3). Intrinsic speed control by multiple receptor types provides the spinal cord with large adaptability and dynamic plasticity. Knowledge of such mechanisms may help to unveil faulty speed control following spinal cord injury (Pepin et al., 2003a,b).

\section{ACKNOWLEDGMENTS}

This work was supported by NIH R01NS040794/08, EU grant (Spinal Cord Repair), Swedish Medical Research Council, and Friends of Karolinska Institutet. We are grateful to Drs. Abdel elManira, Sten Grillner and Kimberley Dougherty for comments on the results and for reading the manuscript, and to Dr. David McCrea for his valuable contribution for the analysis of deletions. We thank Dr. Jesper Ryge for scripts for the R package, and Ann-Charlotte Westerdahl for technical assistance.

glycine receptor. Eur. J. Pharmacol. 156, 177-180.

comotor pattern generation in th neonatal rat spinal cord. Proc. Biol. Sci. 264, 877-884.

Birch, P. J., Grossman, C. J., and Hayes, A. G. (1988). 6, 7-Dinitro-quinoxaline-2, 3-dion and 6-nitro, 7-cyano-quinoxaline-2,3-dion antagonise responses to NMDA in the rat spinal cord via an action at the strychnine-insensitive
Brodin, L., and Grillner, S. (1985). The role of putative excitatory amino acid neurotransmitters in the initiation of locomotion in the lamprey spinal cord. I. The effects of excitatory amino acid antagonists. Brain Res. 360, 139-148.
Brodin, L., Grillner, S., and Rovainen, C. M. (1985). N-Methyl-D-aspartate (NMDA), kainate and quisqualate receptors and the generation of fictive locomotion in the lamprey spinal cord. Brain Res. 325, 302-306.

Butt, S. J., and Kiehn, O. (2003). Functional identification of interneurons responsible for leftright coordination of 
hindlimbs in mammals. Neuron 38, 953-963.

Cazalets, J. R., Borde, M., and Clarac, F. (1995). Localization and organization of the central pattern generator for hindlimb locomotion in newborn rat. J. Neurosci. 15, 4943-4951.

Cazalets, J. R., Sqalli-Houssaini, Y., and Clarac, F. (1992). Activation of the central pattern generators for locomotion by serotonin and excitatory amino acids in neonatal rat. J. Physiol. 455, 187-204.

Childs, A. M., Evans, R. H., and Watkins, J. C. (1988). The pharmacological selectivity of three NMDA antagonists. Eur. J. Pharmacol. 145, 81-86.

Clarke, V. R., Ballyk, B. A., Hoo, K. H., Mandelzys, A., Pellizzari, A., Bath, C. P., Thomas, J., Sharpe, E. F., Davies, C. H., Ornstein, P. L., Schoepp, D. D., Kamboj, R. K., Collingridge, G. L., Lodge, D., and Bleakman, D. (1997). A hippocampal GluR5 kainate receptor regulating inhibitory synaptic transmission. Nature 389, 599-603.

Cohen, A. H., and Wallen, P. (1980). The neuronal correlate of locomotion in fish. "Fictive swimming" induced in an in vitro preparation of the lamprey spinal cord. Exp. Brain Res. 41, 11-18.

Cowley, K. C., and Schmidt, B. J. (1995). Effects of inhibitory amino acid antagonists on reciprocal inhibitory interactions during rhythmic motor activity in the in vitro neonatal rat spinal cord. J. Neurophysiol. 74, 1109-1117.

Cowley, K. C., Zaporozhets, E., Maclean, J. N., and Schmidt, B. J. (2005). Is NMDA receptor activation essential for the production of locomotor-like activity in the neonatal rat spinal cord? J. Neurophysiol. 94, 3805-3814.

Dai, W. M., Egebjerg, J., and Lambert, J. D. (2001). Characteristics of AMPA receptor-mediated responses of cultured cortical and spinal cord neurones and their correlation to the expression of glutamate receptor subunits, GluR1-4. Br. J. Pharmacol. 132, 1859-1875.

Douglas, J. R., Noga, B. R., Dai, X., and Jordan, L. M. (1993). The effects of intrathecal administration of excitatory amino acid agonists and antagonists on the initiation of locomotion in the adult cat. J. Neurosci. 13, 990-1000.

Dubuc, R., Brocard, F., Antri, M., Fenelon, K., Gariepy, J. F., Smetana, R., Menard, A., Le Ray, D., Viana Di Prisco, G., Pearlstein, E., Sirota, M. G., Derjean, D., St-Pierre, M., Zielinski, B., Auclair, F., and Veilleux, D. (2008). Initiation of locomotion in lampreys. Brain Res. Rev. 57, 172-182.

Edmonds, B., Gibb, A. J., and Colquhoun, D. (1995). Mechanisms of activation of muscle nicotinic acetylcholine receptors and the time course of endplate currents. Annu. Rev. Physiol. 57, 469-493.

El Manira, A., Kyriakatos, A., Nanou, E., and Mahmood, R. (2008). Endocannabinoid signaling in the spinal locomotor circuitry. Brain Res. Rev. 57, 29-36.

Endo, T., and Kiehn, O. (2008). Asymmetric operation of the locomotor central pattern generator in the neonatal mouse spinal cord. $J$. Neurophysiol. 100, 3043-3054.

Gillis, G. B., and Biewener, A. A. (2001). Hindlimb muscle function in relation to speed and gait: in vivo patterns of strain and activation in a hip and knee extensor of the rat (Rattus norvegicus). J. Exp. Biol. 204, 2717-2731.

Hägglund, M., Borgius, L., Dougherty, K. J., and Kiehn, O. (2010). Activation of groups of excitatory neurons in the mammalian spinal cord or hindbrain evokes locomotion. Nat. Neurosci. 13, 246-252.

Hinckley, C., Seebach, B., and ZiskindConhaim, L. (2005). Distinct roles of glycinergic and GABAergic inhibition in coordinating locomotor-like rhythms in the neonatal mouse spinal cord. Neuroscience 131, 745-758.

Huettner, J. E., and Bean, B. P. (1988). Block of N-methyl-D-aspartateactivated current by the anticonvulsant MK-801: selective binding to open channels. Proc. Natl. Acad. Sci. USA 85, 1307-1311.

Ireland, M. F., Lenal, F. C., Lorier, A. R., Loomes, D. E., Adachi, T., Alvares, T. S., Greer, J. J., and Funk, G. D. (2008). Distinct receptors underlie glutamatergic signalling in inspiratory rhythm-generating networks and motor output pathways in neonatal rat. J. Physiol. 586, 2357-2370.

Jane, D. E., Lodge, D., and Collingridge, G. L. (2009). Kainate receptors: pharmacology, function and therapeutic potential. Neuropharmacology 56, 90-113.

Jiang, Z., Carlin, K. P., and Brownstone, R. M. (1999). An in vitro functionally mature mouse spinal cord preparation for the study of spinal motor networks. Brain Res. 816, 493-499.

Jordan, L. M. (1998). Initiation of locomotion in mammals. Ann. N Y Acad. Sci. 860, 83-93.

Jordan, L. M., Liu, J., Hedlund, P. B. Akay, T., and Pearson, K. G. (2008) Descending command systems for the initiation of locomotion in mammals. Brain Res. Rev. 57, 183-191.

Kaminski, R. M., Banerjee, M., and Rogawski, M. A. (2004). Topiramate selectively protects against seizures induced by ATPA, a GluR5 kainate receptor agonist. Neuropharmacology 46, 1097-1104.

Katz, P. S., Frost, W. N. (1996). Intrinsic neuromodulation: altering neuronal circuits from within. Trends Neurosci. 19, 54-61.

Kessler, M., Baudry, M., and Lynch, G. (1989). Quinoxaline derivatives are high-affinity antagonists of the NMDA receptor-associated glycine sites. Brain Res. 489, 377-382.

Kiehn, O. (2006). Locomotor circuits in the mammalian spinal cord. Annu. Rev. Neurosci. 29, 279-306.

Kiehn, O., Dougherty, K. J., Hägglund, M. Borgius, L., Talpalar, A., and Restrepo, C. E. (2010). Probing spinal circuits controlling walking in mammals. Biochem Biophys Res Commun 396, 11-18.

Kiehn, O., Johnson, B. R., Raastad, M (1996). Plateau properties in mammalian spinal interneurons during transmitter-induced locomotor activity. Neuroscience 75, 263-273.

Kiehn, O., Quinlan, K. A., Restrepo, C. E., Lundfald, L., Borgius, L., Talpalar, A. E., and Endo, T. (2008). Excitatory components of the mammalian locomotor CPG. Brain Res. Rev. 57, 56-63.

Kjaerulff, O., and Kiehn, O. (1996). Distribution of networks generating and coordinating locomotor activity in the neonatal rat spinal cord in vitro: a lesion study. J. Neurosci. 16, 5777-5794.

Krieger, P., Hellgren-Kotaleski, J., Kettunen, P., and El Manira, A. J. (2000). Interaction between metabotropic and ionotropic glutamate receptors regulates neuronal network activity. J. Neurosci. 20, 5382-5391.

Kudo, N., and Yamada, T. (1987) $\mathrm{N}$-methyl-D,L-aspartate-induced locomotor activity in a spinal cordhindlimb muscles preparation of the newborn rat studied in vitro. Neurosci. Lett. 75, 43-48.

Lafreniere-Roula, M., and McCrea, D. A. (2005). Deletions of rhythmic motoneuron activity during fictive locomotion and scratch provide clues to the organization of the mammalian central pattern generator. $J$. Neurophysiol. 94, 1120-1132.

Lanuza, G. M., Gosgnach, S., Pierani, A., Jessell, T. M., and Goulding, M. (2004). Genetic identification of spinal interneurons that coordinate left-right locomotor activity necessary for walking movements. Neuron 42, 375-386.

Liu, J., and Jordan, L. M. (2005) Stimulation of the parapyramidal region of the neonatal rat brain stem produces locomotor-like activity involving spinal 5-HT7 and
5-HT2A receptors. J. Neurophysiol. 94, 1392-1404.

Loscher, W., and Honack, D. (1994). Effects of the non-NMDA antagonists NBQX and the 2,3-benzodiazepine GYKI 52466 on different seizure types in mice: comparison with diazepam and interactions with flumazenil. $\mathrm{Br}$. J. Pharmacol. 113, 1349-1357.

MacLean, J. N., Cowley, K. C., Schmidt, B. J. (1998). NMDA receptor-mediated oscillatory activity in the neonatal rat spinal cord is serotonin dependent. J. Neurophysiol. 79, 2804-2808.

Mayer, M. L., Ghosal, A., Dolman, N. P., and Jane, D. E. (2006). Crystal structures of the kainate receptor GluR5 ligand binding core dimer with novel GluR5-selective antagonists. J. Neurosci. 26, 2852-2861.

McCrea, D. A., and Rybak, I. A. (2008). Organization of mammalian locomotor rhythm and pattern generation. Brain Res. Rev. 57, 134-146.

Menuz, K., Stroud, R. M., Nicoll, R. A., and Hays, F. A. (2007). TARP auxiliary subunits switch AMPA receptor antagonists into partial agonists. Science 318, 815-817.

Micale, N., Zappala, M., Grasso, S., Puja, G., De Sarro, G., Ferreri, G., De Sarro, A., Toma, L., and De Micheli, C. (2002). Novel potent AMPA/kainate receptor antagonists: synthesis and anticonvulsant activity of a series of 2-[(4-alkylsemicarbazono)-(4-amino-phenyl)methyl]-4,5-methylenedioxyphenyl acetic acid alkyl esters. J. Med. Chem. 45, 4433-4442.

Murthy, V. N., Schikorski, T., Stevens, C. F., and Zhu, Y.(2001). Inactivity produces increases in neurotransmitter release and synapse size. Neuron 32, 673-682.

Nagy, G. G., Al-Ayyan, M., Andrew, D., Fukaya, M., Watanabe, M., and Todd, A. J. (2004). Widespread expression of the AMPA receptor GluR2 subunit at glutamatergic synapses in the rat spinal cord and phosphorylation of GluR1 in response to noxious stimulation revealed with an antigen-unmasking method. J. Neurosci. 24, 5766-5777.

Nanou, E., Kyriakatos, A., Bhattacharjee, A., Kaczmarek, L. K., Paratcha, G., and El Manira, A. (2008). Na+-mediated coupling between AMPA receptors and KNa channels shapes synaptic transmission. Proc. Natl. Acad. Sci. USA. 105, 20941-20946.

Nanou, E., Kyriakatos, A., Kettunen, P., and El Manira, A. (2009). Separate signalling mechanisms underlie mGluR1 modulation of leak channels and NMDA receptors in the network underlying locomotion. J. Physiol. 587, 3001-3008.

Nishimaru, H., and Kudo, N. (2000). Formation of the central pattern 
generator for locomotion in the rat and mouse. Brain Res. Bull. 53, 661-669.

Nishimaru, H., Takizawa, H., and Kudo, N. (2000). 5-Hydroxytryptamineinduced locomotor rhythm in the neonatal mouse spinal cord in vitro. Neurosci. Lett. 280, 187-190.

Ornstein, P. L., Schaus, J. M., Chambers, J. W., Huser, D. L., Leander, J. D., Wong, D. T., Paschal, J. W., Jones, N. D., and Deeter, J. B. (1989). Synthesis and pharmacology of a series of 3- and 4-(phosphonoalkyl)pyridine- and -piperidine-2-carboxylic acids. Potent N-methyl-D-aspartate receptor antagonists. J. Med. Chem. 32, 827-833.

Pepin, A., Norman, K. E., and Barbeau, H. (2003a). Treadmill walking in incomplete spinalcord-injured subjects: 1 . Adaptation to changes in speed. Spinal Cord 41, 257-270.

Pepin, A., Ladouceur, M., and Barbeau, H. (2003b). Treadmill walking in incomplete spinalcord-injured subjects: 2 . Factors limiting the maximal speed. Spinal Cord 41, 271-279.

Perez-Otano, I., and Ehlers, M. D. (2005). Homeostatic plasticity and NMDA receptor trafficking. Trends Neurosci. 28, 229-238.

Procter, M. J., Houghton, A. K., Faber, E. S., Chizh, B. A., Ornstein, P. L., Lodge, D., and Headley, P. M. (1998).
Actions of kainate and AMPA selective glutamate receptor ligands on nociceptive processing in the spinal cord. Neuropharmacology 37, 1287-1297.

Quinlan, K. A., and Kiehn, O. (2007). Segmental, synaptic actions of commissural interneurons in the mouse spinal cord. J. Neurosci. 27, 6521-6530.

Roberts, A., Li, W.C., Soffe, S. R., and Wolf, E. (2008). Origin of excitatory drive to a spinal locomotor network. Brain Res. Rev. 57, 22-28.

Schmidt, B. J., and Jordan, L. M. (2000). The role of serotonin in reflex modulation and locomotor rhythm production in the mammalian spinal cord. Brain Res. Bull. 53, 689-710.

Sokal, R. R., and Rohlf, F. J. (1969). Biometry: The Principles and Practice of Statistics in Biological Research, 3rd Edn. San Francisco, CA: Freeman.

Tazerart, S., Vinay, L., and Brocard, F. (2008). The persistent sodium current generates pacemaker activities in the central pattern generator for locomotion and regulates the locomotor rhythm. J. Neurosci. 28, 8577-8589.

Thiagarajan, T. C., Lindskog, M., and Tsien, R. W. (2005). Adaptation to synaptic inactivity in hippocampal neurons. Neuron 47, 725-737.

Wallen-Mackenzie,A.,Gezelius, H., ThobyBrisson, M., Nygard, A., Enjin, A.,
Fujiyama, F., Fortin, G., and Kullander, K. (2006). Vesicular glutamate transporter 2 is required for central respiratory rhythm generation but not for locomotor central pattern generation. J. Neurosci. 26, 12294-12307.

Whelan, P., Bonnot, A., and O'Donovan, M. J. (2000). Properties of rhythmic activity generated by the isolated spinal cord of the neonatal mouse. $J$. Neurophysiol. 84, 2821-2833.

Wondolowski, J., and Frerking, M. (2009). Subunit-dependent postsynaptic expression of kainate receptors on hippocampal interneurons in area CA1. J. Neurosci. 29, 563-574.

Xu, H., Wu, L. J., Zhao, M. G., Toyoda, H., Vadakkan, K. I., Jia, Y., Pinaud, R., and Zhuo, M. (2006). Presynaptic regulation of the inhibitory transmission by GluR5-containing kainate receptors in spinal substantia gelatinosa. $\mathrm{Mol}$. Pain 2, 29.

Zaporozhets, E., Cowley, K. C., and Schmidt, B. J. (2004). A reliable technique for the induction of locomotorlike activity in the in vitro neonatal rat spinal cord using brainstem electrical stimulation. J. Neurosci. Methods 139, 33-41.

Zaporozhets, E., Cowley, K. C., and Schmidt, B. J. (2006). Propriospinal neurons contribute to bulbospinal transmission of the locomotor com- mand signal in the neonatal rat spinal cord. J. Physiol. 572, 443-458.

Zar, J. H. (1974). Biostatistical Analysis. Englewood Cliffs, NJ: Prentice-Hall.

Zhong, G., Diaz-Rios, M., and HarrisWarrick, R. M. (2006). Intrinsic and functional differences among commissural interneurons during fictive locomotion and serotonergic modulation in the neonatal mouse. J. Neurosci. 26, 6509-6517.

Conflict of Interest Statement: The authors declare that the research was conducted in the absence of any commercial or financial relationships that could be construed as a potential conflict of interest.

Received: 08 April 2010; paper pending published: 07 May 2010; accepted: 12 July 2010; published online: 06 August 2010. Citation: Talpalar AE and Kiehn O (2010) Glutamatergic mechanisms for speed control and network operation in the rodent locomotor CPG. Front. Neural Circuits 4:19. doi: 10.3389/fncir.2010.00019 Copyright (c) 2010 Talpalar and Kiehn. This is an open-access article subject to an exclusive license agreement between the authors and the Frontiers Research Foundation, which permits unrestricted use, distribution, and reproduction in any medium, provided the original authors and source are credited. 